\title{
EL PARADIGMA CONSTITUCIONALISTA DE LA AUTORIDAD JURÍDICA*
}

\author{
María Cristina Redondo \\ Conicet (Argentina). Universidad de Génova (Italia)
}

RESUMEN. En este trabajo discuto algunos de los problemas señalados por Luigi FERRAJOLı en su artículo: "Constitucionalismo principialista y constitucionalismo garantista». En primer lugar, en consonancia con las tesis de FERRAJOLI, intento sostener que el constitucionalismo ha de ser interpretado como el reconocimiento explícito de un nuevo paradigma de autoridad jurídica y de Derecho, y que la teoría jurídica positivista-normativista ofrece la mejor explicación de este nuevo paradigma. En segundo lugar, intento mostrar que un constitucionalismo iusnaturalista o iusrealista serían auto-contradictorios. Estas teorías explican incorrectamente las normas constitucionales que imponen límites sustantivos a la autoridad jurídica (los derechos fundamentales) y asumen tesis que contradicen la propuesta central del constitucionalismo. A lo largo del análisis me concentro en la idea de balance para destacar dos cosas. En primer lugar, que el constitucionalismo garantista de FERRAJOLI implica que estos derechos son normas que están relativamente determinadas por el paradigma vigente en la práctica y que, en tal medida, no se identifican ni aplican mediante balances. En segundo lugar, que dicha tesis es totalmente compatible con reconocer que tales derechos - que devienen parte de los criterios últimos de validez en un sistema constitucionalista-, si bien en modo limitado, admiten una pluralidad de interpretaciones y están potencialmente en conflicto. En tal medida, ellos efectivamente están sujetos a balances.

Palabras clave: constitucionalismo, autoridad, balance de razones.

ABSTRACT. In this article I discuss some of the issues raised by Luigi FERRAJOLI in his work «Constitucionalismo principialista y constitucionalismo garantista». Firstly, in accordance with FERRAJOLI, I try to defend that constitutionalism is to be interpreted as the conscious assumption of a new paradigm regarding legal authority and Law, and that a normativist, positivist legal theory offers the best explanation of this new paradigm. Secondly, I try to show that a natural law constitutionalism or a realist constitutionalism would be auto-contradictory theories. They offer an incorrect explanation of the constitutional norms that impose substantial boundaries on legal authority (fundamental rights) and assume thesis which are incoherent with the central proposal of constitutionalism. The analysis is focused on the idea of balance in order to stress two things. On the one hand, that the kind of constitutionalism that FERRAJOLI defends implies that fundamental rights are norms relatively determined by the paradigm established in the practice and, to that extent, they cannot be identified or applied though balances. On the other hand, that such a thesis is fully compatible with the admission that fundamental rights - which become part of the ultimate criteria of validity in a constitutionalist system-, though marginally, tolerate a plurality of interpretations and are potentially in conflict with each other. To that extent, they do depend on balances.

Keywords: constitutionalism, authority, balance of reasons.

* Fecha de recepción: 10 de enero de 2011. Fecha de aceptación: 7 de febrero de 2011. 


\section{LA TEORÍA CONSTITUCIONALISTA DE FERRAJOLI Y EL POSITIVISMO JURÍDICO}

a teoría constitucionalista y garantista de Luigi FERRAJOLI es una propuesta que ya sea por su alcance y profundidad como por su sensibilidad frente a los problemas de las sociedades democráticas contemporáneas merece una detallada atención. En este trabajo presentaré sólo algunas ideas que tienen su origen en la lectura del texto «Constitucionalismo principialista y constitucionalismo garantista» (en adelante CPCG). Antes de hacerlo quisiera subrayar que la propuesta de FERRAJOLI me impacta en modo singular, como un conjunto de tesis extremamente estimulantes y con las que en la mayor parte de los casos me encuentro de acuerdo en lo sustancial.

Como sabemos, Luigi FERRAJOLI ve en el constitucionalismo un nuevo paradigma de Derecho que se ejemplifica en todo sistema jurídico que incorpore una constitución rígida. Estos sistemas jurídicos corresponden al «Estado constitucional de Derecho» cuyas características más salientes, por lo general, se establecen a través de una comparación con el «Estado legislativo de Derecho». Un primer punto importante es que FERRAJOLI, al afirmar que estamos en presencia de un nuevo paradigma, no entiende que el constitucionalismo esté en competición con, o se agregue a, las dos conocidas concepciones del Derecho: el positivismo y el iusnaturalismo ${ }^{1}$. En contraposición con el modo hoy usual de presentar el constitucionalismo, FERRAJOLI sostiene que éste constituye una ruptura o una novedad con relación a una previa versión del positivismo jurídico. Versión ésta que era apta para explicar los sistemas típicos del «Estado legislativo de Derecho», pero que no logra dar cuenta de aquellos que asumen el ideal de la autoridad sustancialmente limitada por el propio Derecho.

La noción de paradigma es compleja y se usa comúnmente con más de un significado. Al hablar del nuevo paradigma constitucionalista, si entiendo bien, FERRAJOLI se refiere a un conjunto de tesis filosóficas, empíricas, políticas, interpretativas, etc., acerca del Derecho y que están implícitas en una práctica efectivamente vigente. Concretamente, según FERRAJOLI, el nuevo paradigma «equivale a un proyecto normativo» que se declina en un preciso modelo, en una específica teoría y en una determinada propuesta filosófico-política respecto del Derecho. El punto importante es que, en su opinión, el modelo, la teoría y la propuesta filosófica de los que estamos hablando hunden sus raíces en la concepción positivista del Derecho, profundizándola.

\subsection{El nuevo paradigma positivista de Derecho}

¿El modelo que da cuenta de los actuales sistemas jurídicos constitucionalistas es realmente novedoso respecto del modelo ofrecido por el positivismo clásico? Quienes

${ }^{1}$ La lectura que FERRAJOLI ofrece del constitucionalismo se contrapone a la que hacen, por ejemplo, P. COMANDUCCI o M. BARBERIS cuando lo entienden como una teoría del Derecho ulterior y contrastante con la del positivismo y del iusnaturalismo. Cfr. P. COMANDuCCI, Hacia una teoría analítica del Derecho, Madrid, Centro de Estudios Políticos y Constitucionales, 2010, 251-264. M. BARBERIS, Filosofia del diritto. Un' introduzione

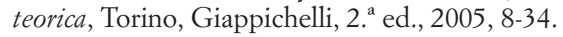


responden que no a esta pregunta sostienen, entre otras cosas, que el paso del Estado legislativo al Estado constitucional de Derecho - momento en el que el cambio de paradigma se hace explícito- es un cambio que puede ser explicado bajo un único modelo positivista de Derecho. En este sentido, los sistemas constitucionalistas contemporáneos, por más diferentes que sean respecto de los sistemas apoyados en la voluntad soberana de un rey o un parlamento tienen idéntica estructura y las mismas características de base. Las diferencias entre los dos sistemas no son cualitativas.

El desacuerdo entre aquellos autores que nieguen y aquellos que como FERRAJOLI sostengan que estamos frente a un nuevo modelo positivista de Derecho podría ser puesto en los siguientes términos. Para los primeros, la diferencia entre un sistema jurídico basado en una autoridad heterónoma soberana (rey, parlamento, congreso, etc.) y uno basado en una autoridad procedimental y sustancialmente limitada por el Derecho reside simplemente en que el último es un Estado que contiene un eslabón más en la cadena legislativa: contiene una ley constitucional. Desde su perspectiva, la teoría clásica del positivismo está en perfectas condiciones para explicar la especial posición de esta ley superior dentro de los sistemas jurídicos.

Aunque por su peculiar importancia la constitución sea fruto de un pacto fundamental, o de un momento político extraordinario, ella no es otra cosa que un ejemplo más de norma jurídica sobre la producción de otras normas jurídicas. Salvo por su específica posición jerárquica, en nada difiere de otras leyes o códigos que establecen órganos competentes (como los que establecen jueces de paz, por ejemplo), regulan el procedimiento de creación de nuevas normas (como los que establecen procedimientos para dictar sentencia) y regulan el contenido de las mismas (como lo hacen el Código Penal o Civil, por ejemplo).

Asimismo, aparentemente, la teoría clásica del positivismo puede explicar con facilidad la primacía o superioridad de aquellas normas constitucionales que limitan a la autoridad y de las que depende la validez de las restantes normas del sistema. En este enfoque, dichas normas procedimentales y sustanciales gozan de prioridad o supremacía porque, y en la medida en que, no pueden ser modificadas por las autoridades cuyos actos controlan, al menos no mediante el procedimiento con que éstas toman sus decisiones ordinarias. En este sentido, la previsión de un específico órgano, o de un procedimiento más exigente que el establecido para la legislación ordinaria, i. e. la imposición de rigidez, es vista como una condición necesaria, o inclusive como una condición necesaria y suficiente de su supremacía. La rigidez es lo que explica por qué las normas en cuestión: $a$ ) son «superiores» o «supremas»; $b$ ) establecen vínculos (procedimentales y sustanciales) a la autoridad, y $c$ ) establecen las condiciones últimas de validez de las restantes normas del sistema. En otras palabras, las normas constitucionales que limitan a la autoridad tienen supremacía si, y sólo si, la propia constitución prevé un procedimiento de modificación agravado para las normas constitucionales.

En la imagen que emerge de este enfoque, en todo sistema jurídico existen, y hay una diferencia crucial entre, autoridades constituyentes (soberanas) y autoridades constituidas (ordinarias). Las autoridades constituidas están limitadas por la ley emanada de la autoridad constituyente. Esta última es quien decide, y establece en una constitución, cuáles son las condiciones últimas para la pertenencia de una norma al sistema. En suma, el paso del Estado legislativo al Estado constitucional de Derecho 
significa que hoy el órgano productor de las leyes ordinarias está sometido a una norma superior. Ahora bien, esta norma superior es fruto de la voluntad de una autoridad positiva última que, ciertamente, puede estar moral o socialmente limitada pero carece de sentido decir que está jurídicamente limitada, puesto que toda norma jurídica depende de ella. Tal como se dijo al inicio, estamos en el mismo esquema sólo que con un eslabón adicional, una autoridad y una norma más en la cadena jerárquica.

En resumen. Conforme a la concepción clásica del positivismo nos encontramos todavía en el modelo, así llamado, del gobierno de la voluntad de los hombres. Es posible restringir cuanto sea imaginable (procedimental y/o sustancialmente) la competencia de las autoridades (constituyentes o constituidas), en cualquier caso, las normas que restringen la competencia son el producto de la voluntad una autoridad constituyente. Este legislador especial mañana podría cambiar opinión y modificarlas según su voluntad $^{2}$.

Para FERRAJOLI, en cambio, no parece ser la restricción al procedimiento de modificación de la constitución la razón de la supremacía de las normas (procedimentales y sustanciales) que limitan a la autoridad, ni del hecho que ellas constituyan criterios últimos de validez jurídica. Por el contrario, es el reconocimiento de que conforme al paradigma instaurado en la práctica ciertas normas procedimentales y sustanciales constituyen un límite a toda autoridad lo que explica que dichas normas sean incorporadas como criterios últimos de validez jurídica y protegidas mediante constituciones rígidas. Es decir, es lo que explica que dichos límites se reconozcan explícitamente en documentos jurídicos y sean resguardados frente a las decisiones de las autoridades, estableciendo procedimientos especiales para su modificación, o inclusive excluyendo totalmente esa posibilidad. En este enfoque, obsérvese, la previsión de un procedimiento agravado para la modificación de las normas constitucionales no es condición ni necesaria ni suficiente de la superioridad de las normas que limitan a la autoridad ${ }^{3}$. En realidad, ellas son supremas en el sistema porque son últimas o no derivadas y esto significa que no pueden ser válidamente creadas, modificadas, o derogadas, siguiendo las normas sobre la producción previstas por el sistema. Ni siquiera a través de procedimientos agravados. Las normas últimas de un sistema, por hipótesis, son independientes y pertenecen al mismo en virtud de factores extra-sistemáticos ${ }^{4}$. En

2 En la teoría positivista clásica es usual concebir esta autoridad a la luz de la noción de soberano propuesta por J. Austin. Existe una extensa discusión acerca de los problemas de auto-referencia, o de regreso al infinito, que plantea el intento de explicar los límites jurídicos de la autoridad así entendida. Al respecto, E. GARZÓN VALDÉS, «Las limitaciones jurídicas del soberano», 1983, en Derecho, ética y política, Madrid, Centro de Estudios Constitucionales, 1993, 181-200.

3 Si entiendo bien, es esta la posición de L. PRIETo y que es objeto de la crítica de J. C. BaYÓn. Según este último, la previsión de un procedimiento de revisión agravado es una condición necesaria, sin la cual no puede decirse que una norma tenga supremacía. Según la posición de PRIETO, la previsión de un procedimiento agravado no es necesaria justamente porque, aunque no exista, está implícita en aquello que significa ser una norma suprema. Al respecto, J. C. BAYÓN, «Democracia y derechos: problemas de fundamentación del constitucionalismo», en J. Betegón, F. LAPORTA, J. R. PÁramo y L. Prieto SAnChís (eds.), Constitución y derechos fundamentales, Madrid, Centro de Estudios Políticos y Constitucionales, 2004, 67-118.

${ }^{4}$ Con respecto a los criterios de validez de normas en un sistema jurídico es imprescindible distinguir entre aquellos cuya aplicación identifica normas independientes y aquellos que identifican normas dependientes. La exigencia de que todas las normas satisfagan los mismos criterios conduce a un regreso al infinito o a un círculo vicioso. Cfr. R. CARACCiolo, El sistema jurídico. Problemas actuales, Madrid, Centro de Estudios Constitucionales, 1988, 31. 
mi opinión, la novedad del constitucionalismo tal como FERRAJOLI lo entiende es que el factor extra-sistemático del que estas normas últimas dependen no es el hecho de que estén impuestas por la voluntad de una autoridad, ni el que sean reconocidas por los órganos de aplicación, sino el que se acepten como justificadas en el paradigma imperante ${ }^{5}$.

Vistas así las cosas, puede afirmarse que el nuevo paradigma recoge la idea del gobierno de la ley sobre la voluntad de los hombres. En esta perspectiva, los criterios últimos de validez de las normas no dependen de la voluntad de una autoridad, sin aditamentos, ni constituyente ni constituida. Ni siquiera si ella es democrática. Tales límites están justificados en el paradigma imperante y son inderogables o indisponibles por parte de la autoridad. Cabe recordar que una autoridad es alguien que decide en lugar de otro ${ }^{6}$. La autoridad jurídica, concretamente, sustituye en sus decisiones a los individuos que están bajo su competencia. Ahora, conforme al actual paradigma, los únicos agentes «soberanos» son los individuos. La autoridad, en cambio, está conceptualmente sujeta a restricciones (procedimentales y sustanciales) que, en la medida en que estén identificadas por el propio Derecho, devienen restricciones jurídicas.

Estas afirmaciones merecen ser precisadas porque, aparentemente, ponen en duda un principio fundamental del positivismo que FERRAJOLI claramente acepta: auctoritas non veritas facit legem. En efecto, la idea de que el Derecho depende en última instancia de una autoridad jurídica puede sugerir que ésta tiene en sus manos la posibilidad de, válidamente, deshacerse de todo límite. Si toda limitación jurídica depende de la voluntad de la autoridad, ¿`cómo es posible, en última instancia, una autoridad jurídicamente limitada? Frente a este viejo problema de la limitación de la autoridad última sólo quisiera subrayar lo siguiente. $\mathrm{Si}$ «auctoritas non veritas facit legem» significa que aquello que es jurídicamente válido está totalmente a disposición de la voluntad de la autoridad, cualquiera sea esta voluntad, entonces llevan razón aquellos autores que sostienen que no estamos frente a un nuevo paradigma ya que todo el Derecho, incluidas las normas últimas que pretenden constituir vínculos a la autoridad, en realidad, dependen de ella. Si el paradigma del constitucionalismo garantista representa una novedad respecto del modo estándar de concebir el Derecho en la óptica positivista, entonces, o bien tenemos que rechazar que auctoritas non veritas facit legem, o bien tenemos que admitir que no puede tener el significado antes indicado. A mi entender, en esta nueva perspectiva, aun cuando toda norma jurídica válida dependa en última instancia de la voluntad de una autoridad, no cualquier voluntad de la autoridad da lugar a una norma válida ${ }^{7}$. Lo hace sólo si respeta ciertas normas independientes, cuya pertenencia al sistema jurídico depende de factores extra-sistemáticos distintos de su propia voluntad.

En este punto, no sé si comprendo bien las tesis de FERRAJOLI. Conforme a específicos postulados de su teoría, que no son discutidos en el trabajo al que me estoy

5 Según C. S. NINO, entre los criterios de individualización de normas no derivadas se encuentran el criterio territorial, el del origen en un cierto legislador, el de la norma fundamental, el de la regla de reconocimiento y el del reconocimiento de los órganos primarios. Cfr. C. S. NiNO, op. cit., 118-131. En mi opinión cabría agregar el criterio de la justificación en el paradigma imperante.

6 Cfr. J. RAz, Practical Reasons and Norms, 1. ${ }^{\text {a }}$ ed., London, Hutchinson, 1995, 62-64.

7 Aun si consideramos al electorado como autoridad última, éste no está libre de limitaciones jurídicas. Cfr. H. L. A. HART, The Concept of Law, 2. ${ }^{a}$ ed., Oxford, Clarendon Press, 78. 
refiriendo, las normas últimas de un sistema dependen en general de un poder constituyente que no es otra cosa que una situación originaria, consistente en una facultad, es decir en un poder, que se caracteriza por ser, no positivo - porque no es producto de ningún acto-y no normativo - porque no es regulado, y que es ejercitado por sujetos naturales-. Éstos, en el caso de los sistemas democráticos, serían todos los miembros de la sociedad, que ejercitándolo dan vida al pactum associationis ${ }^{8}$.

En mi opinión, cabe subrayar que no todas las normas constitucionales son normas últimas del sistema jurídico. Lo son sólo aquellas que establecen las condiciones de validez de las normas en el sistema. Es decir, las que establecen las condiciones (procedimentales y sustanciales) que la autoridad jurídica debe respetar. Estas normas últimas son criterios independientes, y pertenecen al sistema, o bien en virtud de una fuente social — como piensa un positivista-, o bien en virtud de consideraciones morales, i. e. independientes de toda fuente social —como piensa un iusnaturalista- Tertium non datur. Ante esta alternativa, y en consonancia con el positivismo, entiendo que es la práctica instaurada la que establece en última instancia cómo ha de entenderse la autoridad jurídica y cuáles son sus límites. En este sentido, y sobre la base de una idea que el mismo FERRAJOLI nos ofrece, se puede afirmar que las normas últimas de un sistema pertenecen porque se aceptan como justificadas, dentro del paradigma en vigor. Es interesante observar que la práctica en la que se exhibe el paradigma es más amplia y más profunda que aquella que para HART constituye la «regla de reconocimiento» de un sistema. Según FERRAJOLI, hemos llegado al paradigma constitucionalista, como a toda situación social compleja, a través de luchas, avances y retrocesos históricos. En todo caso, cuál sea el paradigma efectivamente establecido es algo que no depende - como en cambio sí depende la regla de reconocimiento hartiana - de la práctica de identificación de normas desarrollada por los jueces u otros órganos de aplicación 9 .

Puede decirse que la vigencia del paradigma constitucionalista no es otra cosa que la efectiva aceptación de la idea del gobierno de la ley sobre la voluntad de los hombres en sustitución de la vieja idea de la autoridad ilimitada. El pacto constitucional es el reconocimiento consciente de esta mutación de paradigma. En resumen, los Derechos que responden al modelo constitucionalista, por una parte, introducen explícitamente la nueva concepción de autoridad. Es decir, incorporan la regla constitutiva vigente en el paradigma: toda autoridad ha de respetar los límites procedimentales y sustanciales establecidos por el Derecho. Por otra parte, tales sistemas intentan formular expresamente los límites a los que, conforme al paradigma, dicha autoridad está sujeta. Es decir, hacen parcialmente explícito, en un pacto constitucional, el contenido del paradigma.

\subsection{Algunos corolarios}

Según como se desee expresarlo, bajo el paradigma constitucionalista, los límites a la autoridad jurídica indican deberes, o ausencia de competencias, que, o bien se

8 Cfr. L. FerRajol, Principia iuris, Teoria del diritto e della democrazia. 1. Teoria del diritto, Roma-Bari, Laterza, 2007, 849-854.

9 Por este motivo, no se presentarían los problemas de circularidad que, como señalaran CARACCIOLO y NINO, sí afectan a otros criterios de individuación de normas independientes. Cfr. C. NINO, Introducción al análisis del Derecho, Buenos Aires, Astrea, 2. ${ }^{a}$ ed., 1984, 118-132, esp. 128. 
aplican a la autoridad jurídica, definida independientemente, o bien son parte de la definición de autoridad jurídica. Conforme a la primera presentación el paradigma constitucionalista puede ser visto como una sustitución del paradigma del gobierno de la voluntad de la autoridad por el del gobierno de la ley sobre la voluntad de la autoridad. Conforme a la segunda presentación, el paradigma constitucionalista es compatible con la idea de que el Derecho depende siempre y exclusivamente de la voluntad de una autoridad. Sólo que, estando al paradigma imperante, se ha modificado nuestra concepción de la autoridad. Ninguna autoridad puede decirse soberana o suprema ${ }^{10}$.

Puestas las cosas de este modo, logramos ver en qué consiste uno de los errores fundamentales en el que cae el que aquí he llamado positivismo clásico (y al que FERRAJOLI llama paleo-positivismo). Éste continúa mirando al Derecho con una lente austiniana, y no logra explicar, en términos positivistas, el hecho de que la autoridad esté jurídicamente limitada, sin que esos límites estén a su disposición. En otras palabras, no logra explicar la tesis central del constitucionalismo. El positivismo entendido en términos austinianos está constreñido a admitir que la tesis central del constitucionalismo sólo puede explicarse en términos no-positivistas. De hecho, quienes aceptan este positivismo «clásico» ven en la nueva concepción constitucionalista un retorno, o un re-florecimiento, del iusnaturalismo. En cambio, si lo dicho hasta aquí es correcto, podemos afirmar que hay una teoría positivista que puede explicar sin dificultad - y sin abandonar la tesis de las fuentes sociales_ - la idea de que hay límites que son constitutivos de lo significa ser una autoridad jurídica y que, por tanto, no están a disposición de dicha autoridad. Tales límites son jurídicos en la medida en que son reconocidos como normas últimas, no derivadas, de un sistema jurídico.

Estas apreciaciones ponen de manifiesto la necesidad de distinguir con claridad entre el paradigma de la autoridad limitada (o del gobierno de la ley) y la específica ley o documento constitucional que, entre otras cosas, recepta el paradigma de la autoridad limitada. Si, como piensa FERRAJOLI, el paradigma del que estamos hablando está efectivamente instaurado en las sociedades contemporáneas, aunque las autoridades puedan mediante ciertos procedimientos previstos (agravados o no) cambiar o abolir el pacto constitucional, no pueden cambiar ni abolir del mismo modo el paradigma. La instauración, cambio, o sustitución de un paradigma acerca del Derecho, aunque sin duda es fruto de acciones humanas, no es producto de una específica acción intencional, ni menos aún de actos jurídico-institucionales de los cuales, por el contrario, es condición de posibilidad. Si el actual paradigma ha de modificarse, como de hecho se modificó el correspondiente al Estado legislativo de Derecho, será como resultado de múltiples factores históricos, políticos, económicos, etcétera.

Asimismo, si el paradigma constitucionalista está instaurado en la práctica, el reconocimiento de esta práctica, para quienes estén conscientemente de acuerdo con ella, será una buena razón para intentar cristalizarla y protegerla mediante documentos jurídicos como son las constituciones. Pero inclusive si la ciudadanía no es consciente del paradigma vigente, el mero hecho de que esté vigente contribuye a explicar causalmente por qué muchas sociedades han llegado a pactos explícitos en los que establecen

10 Conforme a FerRAJOLI «en el Estado constitucional de Derecho no existen poderes soberanos o legibus soluti». Cfr. L. FERRAJOLI, op. cit., 854. 
la concepción de la autoridad constitucionalista ${ }^{11}$. En cualquier caso, esto permitiría explicar por qué, aun cuando el paradigma esté establecido, puede haber casos como el de Gran Bretaña, por ejemplo, en los que se ha decidido no formalizarlo, o custodiarlo mediante un documento constitucional. Y, a la inversa, permite también explicar por qué pueden existir documentos o leyes constitucionales que no receptan el paradigma hoy vigente, como sería el caso de toda constitución flexible. Como he dicho antes, los sistemas jurídicos con constituciones rígidas son sólo un ejemplo paradigmático en el que la concepción constitucionalista de la autoridad jurídicamente limitada se asume explícitamente mediante un pacto formal o ley fundamental. Sin embargo, ello no significa que el paradigma no esté en vigor allí donde no hay un documento formal llamado constitución, o que lo esté en cualquier caso en el que exista una constitución en sentido formal ${ }^{12}$.

Por último, es posible pensar que, conforme al presente enfoque, el rol de la autoridad constituyente es meramente epistémico. Es decir, su función se limitaría a conocer el contenido del paradigma para plasmarlo en un documento constitucional. Sin embargo, nada de lo que he dicho debería conducirnos a esta conclusión. He sostenido, en efecto, que los pactos constitucionales que asumen un conjunto normas procedimentales y de derechos fundamentales como límite a la autoridad jurídica, de hecho, están reflejando la concepción de la autoridad vigente en la práctica. Aun cuando se admita que estos límites están parcialmente determinados por el paradigma y que las autoridades pueden conocerlos, ellos nada dicen acerca del específico diseño institucional en el cual se deben traducir. Es decir, la autoridad constituyente no sólo debe decidir cuáles derechos y procedimientos incorpora, debe también diseñar las específicas instituciones en las cuales ellos se ejercen. Por ejemplo, debe decidir si instaura un sistema de parlamentarista o presidencialista, y de qué tipo concretamente. Si implementa un sistema de control de legitimidad constitucional, y con qué características. En otras palabras, si bien el núcleo conceptual y la posición axiológica de los derechos que limitan a la autoridad no dependen de su decisión, es ella quien decide a través de qué específicas obligaciones, prohibiciones y permisiones implementarlos.

\section{LA CRÍTICA A LA CONCEPCIÓN PREDOMINANTE DEL CONSTITUCIONALISMO}

FERRAJOLI identifica dos tipos de enfoques teóricos que ofrecen una visión inadecuada del nuevo paradigma constitucionalista: por una parte, el post-positivismo junto al iusnaturalismo, que con respecto al tema en discusión sostienen tesis prácticamente idénticas y, por otra parte, el iusrealismo.

${ }^{11}$ Esto no significa que la práctica sea necesariamente precedente a la existencia de constituciones rígidas. Como cuestión de hecho es posible que la existencia de pactos constitucionales rígidos haya contribuido a instaurar el paradigma constitucionalista en la práctica.

12 El contraste entre paradigma constitucionalista y constitución en sentido formal puede sugerir que el paradigma deba ser entendido como la constitución material común a los sistemas jurídicos con constitución formal. Sin embargo, no es así. Al menos no lo es en muchos de los sentidos que se da a la expresión «constitución material», que es extremadamente polivalente. Cfr. R. BIN, «Che cos'è la costituzione?», Quaderni Costituzionali 1, Il Mulino, 2007, 11-52, 35. 
En primer lugar me referiré muy brevemente a algunas apreciaciones de FERRAJOLI que, en su opinión, ofrecen buenas razones para rechazar la conexión necesaria entre Derecho y moral. Al respecto, la posición de FERRAJOLI no parece convincente. A mi entender los argumentos que presenta se basan en un concepto de moral y de objetividad extraños al debate contemporáneo, razón por la cual resulta difícil aplicar su crítica a los post-positivistas y iusnaturalistas que toma en consideración. El mejor argumento para adoptar el método positivista, en cambio, es el que FERRAJOLI presenta cuando muestra que la teoría positivista está en condiciones de dar adecuadamente cuenta de las ideas centrales del constitucionalismo mientras que no puede decirse lo mismo de las teorías que asumen un enfoque iusnaturalista o iusrealista.

En segundo lugar, me referiré concretamente a los argumentos que muestran por qué tanto el enfoque iusnaturalista como el iusrealista fracasan: estas posiciones ofrecen una explicación inadecuada de los derechos fundamentales, que son las normas que imponen límites sustanciales a la autoridad en el paradigma constitucionalista. Esta vez sí creo que FERRAJOLI lleva la razón y que a partir de sus razonamientos podemos afirmar no sólo que las posiciones criticadas tienen una teoría deficitaria respecto de estas normas fundamentales, sino una tesis aún más radical. En efecto, si asumimos que la teoría del constitucionalismo (o al menos su tesis central acerca de la autoridad limitada) es una teoría posible, las propuestas del post-positivismo, iusnaturalismo y iusrealismo deben ser rechazadas por una razón muy convincente: porque asumen tesis que hacen del constitucionalismo una teoría auto-contradictoria. Aun cuando estas posiciones, en abstracto, sean coherentes y ofrezcan un modelo posible de Derecho, no ofrecen una teoría coherente del constitucionalismo. En otras palabras, no es posible un constitucionalismo iusnaturalista o un constitucionalismo realista.

Como bien observa FERRAJOLI, al caracterizar la identificación y la aplicación de las normas que expresan derechos fundamentales, tanto iusnaturalistas como iusrealistas apelan a la idea de balance o ponderación. Es decir a un tipo de razonamiento en el que se cotejan aquellas consideraciones que resultan relevantes a fin de obtener, todas las cosas consideradas, una conclusión ${ }^{13}$. Concretamente, respecto de la identificación de los derechos jurídicamente válidos y/o aplicables, el balance o ponderación, o bien es el nombre del mismo proceso de interpretación, o bien el de un específico razonamiento interpretativo, indispensable para dicha identificación. En cualquier caso, quien sostiene que la identificación o interpretación de los derechos requiere o consiste en un balance está diciendo que el contenido de los derechos válidos y/o aplicables es el producto de una ponderación y está supeditado a la consideración de todo aquello que resulte relevante en el razonamiento mediante el que dicho contenido se identifica.

A su vez, respecto de la aplicación de los derechos fundamentales, el balance, o bien es el nombre del propio proceso de aplicación, o bien el de un específico razonamiento indispensable para obtener una conclusión deóntica. Quien sostiene que la aplicación de estos derechos requiere o consiste en un balance está diciendo que las normas que establecen derechos fundamentales no permiten por sí solas separar una conclusión deóntica respecto de una acción o, lo que es lo mismo, no se aplican me-

13 El balance no es un razonamiento en sentido lógico. La conclusión que en él se extrae no está determinada por reglas de inferencia lógica, sino por la importancia o fuerza relativa de los datos evaluados como relevantes. 
diante una subsunción. Su aplicación, o bien requiere necesariamente, o bien consiste en una evaluación en la que ellos se comparan con todas aquellas otras consideraciones que resulten relevantes en la ocasión de aplicación. Sólo así podremos determinar cuál tiene más peso y determina aquello que, todo considerado, se debe hacer.

Como veremos más adelante, son éstas las ideas responsables del error en el que caen las teorías de corte iusnaturalista y iusrealista, puesto que éstas son las ideas que tornan incoherente la tesis central del constitucionalismo.

\section{1. ¿Un constitucionalismo iusnaturalista?}

Las lecturas iusnaturalistas y post-positivistas están de acuerdo en que, respecto del modelo del Estado legislativo de Derecho, el constitucionalismo involucra un cambio paradigmático en la concepción de la autoridad jurídica y del Derecho. Ahora bien, ellas entienden que el nuevo paradigma requiere el abandono, o cuando menos la modificación, de la teoría positivista.

\subsubsection{La crítica a la conexión entre Derecho y moral}

En opinión de FERRAJOLI no hay ningún problema en reconocer que las normas que expresan derechos fundamentales, como muchas otras del ordenamiento jurídico, tienen un contenido moral, o expresan una pretensión (la de sus autores) de justicia. El problema radica, según FERRAJOLI, en que el iusnaturalismo y el post-positivismo entienden que las normas y valores incorporados, o a los que el Derecho remite, son parte de una moral objetiva, verdadera. Es decir, el defecto fundamental de estas teorías es que están asumiendo el objetivismo y el cognoscitivismo éticos. Al hacerlo, estarían admitiendo «...de modo inevitable, el absolutismo moral y, consiguientemente, la intolerancia ante las opiniones morales disidentes» (CPCG). No es éste el lugar en el que se pueda discutir con profundad esta equiparación, a mi juicio problemática, entre las tesis meta-éticas del objetivismo y el cognoscitivismo éticos y las tesis de una ética normativa iliberal e intolerante. En todo caso, esta forma de entender la moral objetiva y el cognoscitivismo no es la que explícitamente manejan autores como DWORKIN, AtienZA, Alexy o Moreso, a quienes él se refiere en sus críticas.

Asimismo, FERRAJOLI sostiene por ejemplo que «una ética objetiva es, inevitablemente, una ética heterónoma...» (CPCG) razón por la cual, y del todo consecuentemente, sostiene también que «no debemos confundir el objetivismo y el cognoscitivismo con la argumentación racional: la solución de una cuestión ética o política que argumentamos como racional no es más "verdadera" que la solución opuesta» (CPCG). En mi opinión es un error caracterizar el objetivismo y el cognoscitivismo moral como posiciones no-racionalistas o, más aún, en contraste con lo que propondría una teoría de la argumentación racional aplicada a la moral. La caracterización que ofrece FERRAJOLI conduce a la conclusión de que la tesis de la conexión entre Derecho y moral objetiva significa conexión entre Derecho y decisiones no racionalmente justificadas. Bajo esta presuposición creo que todos los autores iusnaturalistas y post-positivistas que FERRAJOLI menciona estarían de acuerdo con él en que la tesis de 
la conexión entre Derecho y moral objetiva es incompatible con el constitucionalismo y no debería ser adoptada. Conforme a la mayor parte de la literatura contemporánea el objetivismo y el cognoscitivismo en moral no sólo son compatibles sino que se basan en la argumentación racional. Es decir, las normas de la moral objetiva son aquellas normas apoyadas por las mejores razones, o aquellas a las que llegamos mediante un razonamiento adecuado. Si por un momento admitimos esta tesis, ¿qué es lo que diferenciaría a la posición de FERRAJOLI de la del iusnaturalismo? En realidad, bajo esta hipótesis, parecen ser idénticas.

Un ulterior argumento a favor de la tesis metodológica positivista, que pone a la moral conceptualmente fuera del Derecho es que, si no procedemos de este modo, perdemos la especificidad de la crítica que las normas jurídicas constitucionales permiten formular respecto de normas jurídicas ordinarias, confundiéndola con una crítica moral, externa al Derecho. En efecto, el parámetro de evaluación que las constituciones introducen al incorporar derechos fundamentales hace posible juzgar el Derecho que «es» sobre la base de lo que él, en virtud del pacto constitucional, «debe ser». Siendo ésta una evaluación jurídica, interna al Derecho. En este sentido, si asumimos la tesis positivista que propone FERRAJOLI, es posible que una decisión sea constitucionalmente ilegítima y no por ello sea moralmente criticable y, a la inversa, podría ser moralmente criticable pero no constitucionalmente ilegítima. Este es un punto ciertamente relevante pero, en mi opinión, las teorías post-positivistas e iusnaturalistas pueden acomodarlo sin ninguna dificultad. Si las normas que expresan derechos fundamentales son o remiten a pautas morales, se sigue que la crítica de ilegitimidad constitucional basada en dichas normas es un tipo de crítica moral. Sin embargo, ello no comporta que el Derecho, incluidas sus propias normas constitucionales, no pueda ser objeto de críticas morales adicionales, desde una perspectiva externa a él ${ }^{14}$. Es decir, aunque el Derecho incorpore pautas morales objetivas es posible distinguir los requerimientos morales que están incorporados al Derecho de aquellos que no lo están ${ }^{15}$. De este modo, es posible mantener diferenciados los dos tipos de evaluación.

La mayor parte de los reproches que FERRAJOLI formula acerca de la conexión Derecho-moral está condicionada por el concepto de moral que está usando. Como vimos, cuando FERRAJOLI se refiere a la moral la entiende como una esfera de consideraciones que establecen aquello que es verdadero o correcto en términos absolutos y que, por tanto, se deben concluyentemente obedecer. Para las actuales posiciones iusnaturalistas y post-positivistas, en cambio, que algo sea una consideración moral no significa que ella establezca aquello que es correcto en modo absoluto, ni que concluyentemente se deba obedecer ${ }^{16}$. Por ejemplo, puede ser correcto dejar de lado los requerimientos morales incorporados al Derecho si existen razones morales más importantes, externas al Derecho, que así lo exigen. En suma, afirmar que el Derecho

${ }_{14}$ Por ejemplo, R. Alexy, Teoría de la argumentación jurídica, trad. M. AtIENZA e I. EsPejo, Madrid, Centro de Estudios Constitucionales, 1989, 36, 208, 274, 316.

15 Cfr. R. Dworkin, «The Moral Reading of the Constitution», The New York Review of Books, March, 21, 1996, 46-50, esp. 48.

${ }_{16}$ En rigor, esta idea respecto de la moral la aceptan no sólo las teorías iusnaturalistas, también la acepta el mayor exponente del así llamado «positivismo excluyente», Joseph RAZ. Cfr. J. RAZ, «The Argument from Justice, or How not to Reply to Legal Positivism», G. PAvlakos (ed.), Law, Rights and Discourse. The Legal Philosophy of Robert Alexy, Oxford and Portland, Oregon, Hart Publishing, 2007, 1-17. 
incluye normas morales o necesariamente remite a ellas, en ningún caso implica que se pierda la posibilidad de diferenciar con claridad la crítica de inconstitucionalidad de otras críticas morales, o que se siga el deber concluyente de obedecer al Derecho.

\subsubsection{La crítica a la explicación de las normas que confieren derechos fundamentales}

$\mathrm{El}$ argumento, a mi entender decisivo, en contra de las posiciones post-positivistas e iusnaturalistas se encuentra en la crítica que FERRAJOLI formula respecto de la forma en que estas posiciones configuran las normas constitucionales que confieren derechos. En efecto, la comprensión que tengamos de este tipo de normas es crucial puesto que sobre ellas se sostiene el núcleo de propuesta constitucionalista: ellas son las que limitan a la autoridad imponiéndole prohibiciones y obligaciones.

Sobre este punto parece configurarse un auténtico desacuerdo. Según las posiciones post-positivistas e iusnaturalistas, son justamente las normas que expresan derechos fundamentales las que (al establecer una conexión entre Derecho y moral) requieren para su explicación una teoría superadora del positivismo. Según FERRAJOLI, es justamente esta explicación de corte iusnaturalista la responsable de que tales teorías no puedan dar cuenta correctamente de la tesis central del paradigma constitucionalista y del rol que en ella juegan los derechos fundamentales.

Conforme a las teorías post-positivistas y iusnaturalistas las disposiciones que establecen derechos fundamentales expresan principios derrotables y abiertos. Ellos no determinan deónticamente un comportamiento, sólo contribuyen a hacerlo. Es decir, aunque estas posiciones no lo adviertan, o inclusive lo nieguen explícitamente, lo dicho presupone que las disposiciones que establecen derechos fundamentales expresan razones ordinarias para la acción. Sobre este tipo de razones me interesa sólo subrayar lo siguiente. Ellas están ligadas a la idea de balance en el doble sentido antes señalado: se identifican y se aplican mediante un balance. Las razones válidas y/o aplicables a favor o en contra de una acción no son otra cosa que aquellas consideraciones que resultan relevantes en el razonamiento mediante el que se responde a la pregunta acerca del por qué debo realizar o abstenerme de realizar una acción. Asimismo, las razones ya identificadas se aplican mediante un razonamiento en el que se compara su «peso» o «relevancia» para establecer cuáles son «vencedoras» y cuáles «derrotadas». Es decir, para establecer, todo considerado, qué se debe concluyentemente hacer.

Las normas son también razones para hacer algo, pero de un tipo especial. A diferencia de las razones ordinarias, las normas sí determinan la calificación deóntica de una acción ante ciertas circunstancias. Por este motivo, si se verifican las circunstancias apropiadas, como bien subraya FERRAJOLI, se aplican en modo subsuntivo. Aun cuando sean muy genéricas o vagas, como lo son muchos principios, siempre permiten separar una consecuencia deóntica respecto de una acción y excluyen la relevancia de cualquier otra consideración que no haya sido prevista como condición de aplicación.

Las normas tienen dos características que no siempre son debidamente subrayadas y que, a mi juicio, son dos caras de la misma moneda. En primer lugar, ellas son válidas sólo con relación a un sistema. En segundo lugar, si lo son, son necesariamente excluyentes. Que una norma es válida sólo en el interior de un sistema significa que toda 
norma válida es, necesariamente, o bien una norma independiente, o bien una norma dependiente. Es válida en un sistema si satisface los criterios (sistemáticos o extrasistemáticos) de pertenencia al mismo y son sensibles necesariamente frente a toda consideración que sea relevante conforme a los criterios del sistema. Que toda norma válida es excluyente significa que dichas normas han de ser identificadas exclusivamente sobre la base de los criterios de un sistema. Es decir, sus condiciones de aplicación y su consecuente deóntico no están abiertos y son sensibles sólo frente a aquellas consideraciones que sean relevantes conforme a los criterios del sistema.

La tesis del constitucionalismo es que los derechos fundamentales son normas válidas últimas que expresan las condiciones (sustanciales) de validez de todas las restantes normas del sistema jurídico. En otras palabras, ellos son concebidos como normas independientes del sistema, cuya satisfacción es necesaria para que cualquier otra norma, dependiente, pueda ser creada o inferida válidamente. En este sentido, son parte de los criterios específicos de validez de los sistemas que los incluyen.

Los autores que califican a los principios constitucionales como normas abiertas que se identifican y/o aplican mediante balances y no admiten la distinción entre normas y razones ordinarias tal como la he reseñado aquí. Ellos sostienen que, en realidad, existen dos tipos de normas. Por una parte, las reglas, que son excluyentes y admiten aplicación subsuntiva. Por otra parte, los principios, que son normas abiertas y derrotables que se identifican y/o aplican mediante balances. Es fácil ver que, según la caracterización que he ofrecido antes, toda norma, sea ella una regla o un principio, respeta un modelo de aplicación subsuntivo y excluyente. Mientras que, conforme a la caracterización apenas mencionada, sólo las reglas obedecen a estos parámetros. Los principios funcionan como razones ordinarias que se sopesan junto a todo aquello que es relevante en las específicas circunstancias en las que se interpretan o aplican. Si los derechos fundamentales son pautas de este tipo, ellos no son aptos para cumplir el rol de criterios de validez de un sistema. Son sólo consideraciones derrotables a favor o en contra de la validez o aplicabilidad de otras consideraciones, también derrotables. Las «normas» válidas, así identificadas, en rigor, no conforman un sistema, constituyen más bien un mero conjunto de razones ${ }^{17}$.

A juicio de FERRAJOLI, esta caracterización de los principios en la que se apoya el constitucionalismo principialista es la responsable del «debilitamiento de la normatividad de las constituciones» (CPCG). La justificación de este diagnóstico es del todo obvia. Si los principios que establecen derechos fundamentales se identifican y/o aplican mediante balances, parece una ironía afirmar que ellos son criterios de validez o imponen límites normativos a la autoridad. Es más, como ya he anticipado, creo que a este respecto las apreciaciones de FERRAJOLI se pueden radicalizar. Bajo esta lectura de los principios constitucionales se banaliza completamente el contraste entre la concepción de la autoridad del Estado legislativo y la del Estado constitucional de Derecho. Si los límites que impone la constitución se identifican y/o aplican mediante balances cabe subrayar que éste es el mismo tipo de límite que rige sobre las decisiones del rey o del parlamento en el Estado legislativo de Derecho y, más aún,

$17 \mathrm{Al}$ respecto, vid. M. BARBERIS, Filosofia del Diritto. Un'introduzione teorica, Bologna, Giappichelli, 3. ${ }^{a}$ ed., 2008, 176. 
es el mismo tipo de exigencia que se aplica a cualquier agente dotado de razón ${ }^{18}$. La metáfora del balance o la ponderación reconstruye la estructura del razonamiento de cualquier agente racional, por este motivo resulta cuanto menos sorprendente conjeturar que la misma idea permita dar cuenta de la exigencia a la que está sujeta la autoridad cuando decimos que está sustancialmente limitada mediante las normas de una constitución rígida. Explicar las normas que limitan a la autoridad como principios, y el razonamiento basado en principios como un razonamiento abierto que se desarrolla mediante un balance o ponderación es tanto como reconocer que no hay una real diferencia entre la concepción de la autoridad del paradigma constitucionalista y la del Estado legislativo de Derecho. Salvo que se piense que las autoridades en este último caso no son agentes racionales sujetos a la normal exigencia de decidir sopesando y ponderando razones.

Coincido con FERRAJOLI en que no hay diferencia estructural entre distintos tipos de normas. Toda norma (sea ella una regla o un principio, independiente o dependiente) se aplica mediante subsunción, y ni su validez ni su específica relevancia se deciden mediante balances abiertos. Esto no significa que no haya ocasiones en las que las autoridades de un sistema jurídico tengan necesariamente que decidir mediante balances abiertos (por ejemplo en los casos de conflictos entre derechos fundamentales). Pero esto es equivalente a decir que, si no hay criterios, las autoridades deben deciden conforme a un juicio discrecional. Si una de las ideas en que se basa el constitucionalismo es que los derechos fundamentales son criterios últimos que restringen la discrecionalidad de la autoridad jurídica imponiéndoles prohibiciones y obligaciones, la última caracterización que deberíamos esperarnos de estos derechos es en términos de pautas abiertas a balances o ponderación. Es un compromiso conceptual del constitucionalismo rechazar esta caracterización. Si no lo hace se auto-contradice, ya que a través de ella estaría negando aquello que afirma como punto de partida: que en el paradigma constitucionalista la autoridad no es meramente un agente racional que decide en modo ponderado, es un agente que se encuentra limitado por normas jurídicas últimas, que no están a su disposición ${ }^{19}$.

18 Sólo por dar algunos ejemplos notables en la literatura, DAVIDSON sostiene que un principio aceptado por todo ser racional es aquél según el cual se debe realizar aquella acción que juzgamos mejor sobre la base de las razones relevantes disponibles (principio de continencia). Cfr. D. DAVIDSON, «How is Weakness of the will Possible?», 1970, Essays on Actions and Events, Oxford, Clarendon Press, 1980, 41. También R. NozICK ve en el balance la estructura del ordinario razonamiento moral. Cfr. R. NozICK, Philosopbical Explanations, Cambridge, Mass., Harvard U. P., 1981, 474-494, esp. 479-482. Asimismo J. RaZ ve el balance como un primer principio de racionalidad (P1). Cfr. J. RAZ, op. cit., 15-48, esp. 36.

19 Este argumento en contra del modo en que las posiciones post-positivistas y iusnaturalistas entienden las normas que expresan derechos fundamentales, en principio, es totalmente independiente de la decisión metodológico-conceptual de considerarlas, o no, pautas morales. El debilitamiento de su normatividad no se produce en virtud de que tales normas tienen carácter moral, sino en virtud de su caracterización como pautas abiertas, sujetas a balances. En esta línea, cabría conjeturar que las teorías de corte iusnaturalista podrían evitar el reproche sosteniendo que contamos con un conjunto último de criterios de validez y aplicabilidad moral que delimitan el sistema de la moral, excluyendo todas aquellas normas que no respeten tales criterios. Desafortunadamente, si es ya altamente controvertido sostener que tales criterios existen respecto de instituciones sociales como son los específicos sistemas jurídicos parece claramente infundado sostener que existen respecto de la moral. En mi opinión, éste no es un defecto de la moral sino justamente un indicador de que aquello que consideramos moral no es un sistema, no está demarcado por un conjunto de criterios últimos sino que depende de lo que se pueda argumentar como relevante, i. e. como válido y/o aplicable, a través de balances de razones. 


\section{2. ¿Un constitucionalismo realista?}

Como bien subraya FERRAJOLI, respecto de la caracterización de los derechos fundamentales como principios, y la de los principios como pautas que se identifican y aplican mediante balances, se configura una convergencia entre las posiciones de corte iusnaturalista y realista. El mismo modelo que bajo presuposición de la existencia de una moral objetiva lleva a conclusiones iusnaturalistas es el que, bajo la presuposición de su inexistencia, lleva a conclusiones iusrealistas. En el primer caso, el resultado del balance depende de lo que es objetivamente relevante en cada situación de interpretación o aplicación, en el segundo, depende de la ponderación del intérprete o aplicador.

En una perspectiva realista, los problemas de interpretación de los derechos fundamentales son ineludibles porque, aun cuando se rechacen tesis radicalmente escépticas respecto del significado en general, las disposiciones que expresan derechos fundamentales, específicamente, y en virtud de su carácter general y controvertido, son siempre interpretables en más de un sentido ${ }^{20}$. Por este motivo, las normas que ellas expresan, i. e. los casos que regulan y las soluciones que establecen, dependen de un razonamiento abierto y, en última instancia, de quién las interpreta. Éste es el punto fundamental en el que el realismo se separa de toda posición positivista-normativista. Según el realismo, las «normas» jurídicamente válidas y aplicables no se identifican mediante criterios que estructuran un sistema de normas, se identifican mediante razonamientos interpretativos, es decir, balances ${ }^{21}$.

Asimismo, una vez resueltos los problemas de interpretación son también ineludibles los problemas ligados a la aplicación, puesto que los derechos fundamentales pueden encontrarse en conflicto y ante la ausencia de criterios para resolverlos, será necesario hacer un balance para determinar cuál es axiológicamente más importante en una determinada ocasión. Nuevamente aquí las posiciones realistas se distancian del positivismo normativista, no porque este último no pueda admitir conflictos entre derechos fundamentales sino porque, al rechazar la existencia de criterios últimos de validez que estructuren un sistema excluyente, los realistas admiten que los principios constitucionales pueden entrar en conflicto con cualquier consideración que a través de un razonamiento adecuado podamos presentar como relevante y aplicable a la situación. En suma, aun cuando las posiciones realistas subrayen que los balances que determinan el contenido o resuelven los conflictos entre derechos no son totalmente abiertos sino que están restringidos por los «textos dotados de autoridad», o se limitan a la consideración de «datos jurídicamente relevantes», el problema es que, en este enfoque, lo que los textos dotados de autoridad dicen, o lo que es jurídicamente relevante, depende siempre de razonamientos abiertos: de la posibilidad de aportar un argumento que se acepte como convincente.

${ }^{20}$ Con referencia específica a los derechos fundamentales, cfr. B. CELANO, «Diritti, principi e valori nello Stato costituzionale di diritto: tre ipotesi di ricostruzione», Analisi e diritto, 2004, 53-74.

21 Como sostiene M. BARBERIS, en este tipo de modelo, a diferencia de aquellos en los que la pertenencia de las normas se basa en criterios (por ejemplo, de legalidad y deducibilidad): «...Una norma pertenece al sistema jurídico [...] no sólo por deducción o delegación, sino también por universalización, especificación analogía o cualquier otra justificación que los juristas consideren suficiente». En este modelo, a red, el Derecho es un conjunto de razones ordinarias. Cfr. M. BARBERIS, op. cit., 176-177. 
En rigor, en virtud de las tesis que acepta, es imposible que el realismo dé cuenta de las normas jurídicas que limitan a la autoridad. Me explico. Todos los conceptos a los que llamamos «valorativos» o «normativos» -incluido el propio concepto de norma - son ambiguos en un modo específico. Por una parte, todos ellos pueden ser entendidos en modo reduccionista, como conceptos meramente descriptivos. Como, por ejemplo, cuando se dice que «obligatorio» es aquel comportamiento cuya no-realización será probablemente sometida a una sanción, o que «justo» es aquello que impone el más fuerte. Sin embargo, y contemporáneamente, todos estos términos pueden ser también interpretados en modo no-reduccionista, como conceptos que incorporan o incluyen un ideal. Como, por ejemplo, cuando se afirma que «obligatoria» es aquella conducta que se apoya en las mejores razones, o que «justo» es aquello que sería aceptado por individuos racionales en una situación hipotética de diálogo. Ciertamente, los hablantes pueden aclarar en qué sentido están usando las palabras y, de ese modo, cancelar toda ambigüedad respecto de lo que ellos dicen. Sin embargo, no está a su disposición cancelar la ambigüedad existente fuera de ese contexto de uso. Es decir, los otros significados, si existen, no pueden ser abolidos por decisión del hablante. El realismo es una posición que ha insistido mucho en esto. Dada la inevitable ambigüedad del lenguaje, en toda ocasión en la que le atribuyamos un significado, en un modo o en otro, conservador o radical, estamos necesariamente optando por una entre las varias alternativas interpretativas existentes ${ }^{22}$.

Esta tesis que atribuye al intérprete la responsabilidad por la inevitable elección que opera es, obviamente, también aplicable a los mismos realistas. Entre las dos interpretaciones efectivamente existentes de las expresiones normativas - y de la expresión «norma», en particular- el realismo escoge la interpretación reduccionista. A tenor de la misma, una norma no es otra que aquello que las autoridades identifican cuando interpretan las disposiciones jurídicas, o cuando deciden sobre su base los casos concretos. De este modo, las así llamadas «normas» carecen de toda dimensión ideal, ellas no prescriben absolutamente nada que vaya más allá de lo que efectivamente dicen quienes las interpretan o aplican. Para ser exactos, en realidad, los realistas pueden aceptar que las normas son entidades ideales o expresan significados ideales. El problema, en este caso, es que estos ideales no se aplican solos. En el ámbito jurídico se aplican necesariamente a través de autoridades ${ }^{23}$. Como se ve, en todo caso, la concepción realista hace imposible expresar la idea de que las normas limitan a las autoridades.

En resumen, lo que me interesa destacar son dos cosas, que reitero. En primer lugar, la interpretación reduccionista que el realismo adopta respecto del concepto de norma no es ineludible. La opción reduccionista es sólo una de las lecturas existentes de los conceptos normativos en general, y del concepto de norma en particular. Doy por sentado que el realismo acepta esta conclusión, dado que en caso contrario estaría diciendo que hay atribuciones de significado verdaderas y falsas, y que su interpretación reduccionista, a diferencia de la no-reduccionista, no es opcional, porque capta el verdadero significado de los términos normativos. Esto, como mínimo, sería paradójico.

22 Cfr. P. ChIAssoni, Tecnica dell'interpretazione giuridica, Bologna, Il Mulino, 2007, 141.

23 Cfr. R. GuAstini, L'interpretazione dei documenti normativi, Milano, Giuffrè, 2004, 13. 
En segundo lugar, la interpretación reduccionista del realismo torna lógicamente imposible o contradictoria la afirmación según la cual las autoridades están limitadas por normas. En este sentido, un constitucionalismo realista sería una posición internamente incoherente. Por una parte afirmaría que las autoridades están limitadas por normas - que es una tesis central del constitucionalismo- pero, por otra parte, que las normas, por sí solas, no pueden limitar a las autoridades puesto que ellas necesitan de las autoridades para ser identificadas y aplicadas - que es una tesis central del realismo- - Si éste es nuestro modo de entender las normas que expresan derechos fundamentales lo que estamos haciendo no es ofrecer una interpretación o una versión del constitucionalismo, sino quitar sentido a su premisa fundamental.

\section{3. ¿SISTEMAS JURÍDICOS PLURALISTAS SIN CONFLICTOS?}

La expresión «conflicto entre normas» puede entenderse en modos diferentes. Si adoptamos la idea de ALCHOURRÓN y BULYGIN, por ejemplo, podemos admitir que un conflicto se presenta cuando, con relación a un caso, un sistema normativo ofrece dos o más soluciones, de tal manera que la conjunción de dichas soluciones es una contradicción deóntica ${ }^{24}$. En este caso, es imposible satisfacer el contenido deóntico de ambas normas. El conflicto, así entendido, supone la existencia de una antinomia que torna incoherente el sistema jurídico en el que se presenta.

En la teoría de FERRAJOLI, y conforme a su definición de antinomia, no existen antinomias entre derechos fundamentales ${ }^{25}$. Sin lugar a dudas su definición consiente que ellas existan entre normas (procedimentales y sustanciales) sobre la producción jurídica y normas ordinarias, sin embargo, «los límites estructurales provenientes de algunos derechos al ejercicio de otros $[\ldots]$ no darán lugar a conflictos ni a balances» (CPCG).

Distintos autores se han detenido, por ejemplo, en la hipótesis de «conflictos» entre derechos del mismo tipo imputables a dos o más sujetos diferentes ${ }^{26}$. Estas situaciones representan evidentemente la existencia de un conflicto en algún sentido del término, pero no refieren necesariamente a la pertenencia de dos normas antinómicas en un sistema jurídico. Así, cuando un juez afronta el «conflicto» en el que debe decidir entre el derecho a la vida de Juan y de Pedro porque en virtud de las circunstancias individuales, respetar el derecho del primero implica abstenerse de respetar el del segundo, no se encuentra ni siquiera ante dos normas diferentes, menos aún antinómicas. Para sostener que hay una antinomia deberíamos decir que todas las normas individuales, tales como las que imponen el deber de respetar la vida de Juan y el deber de abstenerse de hacerlo en una ocasión específica, pertenecen

${ }^{24}$ Cfr. C. AlchourRón y E. Bulygin, Introducción a la metodología de las ciencias jurídicas y sociales, Buenos Aires, Astrea, 1975, 101.

25 D10.43: «Antinomia» è il vizio sostanziale prodotto dall' indebita decisione di una noma in contrasto con una norma sostanziale sulla produzione, la cui applicazione suppone l'annullamento della norma in contrasto. Cfr. L. FERRAJOLI, op. cit., tercer volumen, 508.

${ }^{26}$ Los ejemplos a los que me refiero pueden verse en el trabajo de G. PINO, quien, efectivamente, los entiende como ejemplos de conflictos entre derechos fundamentales. Cfr. G. PINO, «Conflictos entre derechos fundamentales. Una crítica a Luigi Ferrajoli», Doxa, 2010, 657-660. 
al sistema. Pero ésta es una reconstrucción ad hoc, claramente insatisfactoria, y que tendría como única justificación el poder sostener que efectivamente pertenecen al sistema dos normas individuales que son antinómicas. Algo parecido sucede ante el derecho a alimentarse de un individuo (entendido como inmunidad) y el derecho a la vida de otros (que podrían beneficiarse con la abstención del primero, usando los recursos liberados para conservar su vida). O ante dos o más derechos sociales, cuando la escasez de recursos materiales impide satisfacer ambos. Sin duda afrontamos un importante problema, y hasta quizás un dilema moral, si habiendo reconocido el derecho a la educación y el derecho a la salud contamos con recursos tan magros que alcanzan sólo ocasionalmente para honrar parcialmente a uno de ellos. Pero estas situaciones no necesariamente indican que el sistema jurídico contiene normas contradictorias. En otras palabras, no hay ninguna razón teórica que imponga la necesidad de reconstruir estas situaciones como una antinomia entre normas que deben ser sometidas a un balance o ponderación. De hecho, FERRAJOLI las configura como hipótesis en la que la aplicación de las normas involucradas requiere sí una ponderación, pero de aspectos empíricos. En su opinión, «la ponderación se produce en cualquier actividad jurisdiccional donde se dé el concurso de varias normas diversas, sean reglas o principios. Pero tiene por objeto no las normas a aplicar, sino, antes bien, las circunstancias de hecho previstas por las mismas» (CPCG). Este tipo de ponderación se presenta siempre, y no sólo en la aplicación de derechos fundamentales. En el Derecho penal, por ejemplo, cabe considerar el balance «entre las circunstancias agravantes y circunstancias atenuantes del delito», $\mathrm{o}$ «...la ponderación que requiere la valoración de circunstancias eximentes, como el estado de necesidad o la legítima defensa» (CPCG). En suma, FERRAJOLI puede correctamente configurar estas situaciones en modo tal que ellas no impliquen la existencia de una antinomia entre las normas pertenecientes al sistema.

Una vez precisadas las circunstancias empíricas de aplicación podríamos advertir que en el sistema existen soluciones normativas precisas, claramente ordenadas. Por ejemplo, respecto de los aparentes conflictos entre derechos de distinto tipo, como el derecho a la intimidad o al honor y el derecho a la información o la libertad de expresión. Si se precisa que estamos hablando del derecho a la intimidad de altos funcionarios del Estado, es posible que sea claro que, para ese tipo de casos, en el sistema prevalezca el derecho a la información. Sin embargo, esto es contingente y, si los criterios de ordenación no permiten establecer qué derecho prevalece, entonces es posible que el sistema que sólo prevé en abstracto el derecho a la intimidad y el derecho a la información contenga una antinomia implícita ${ }^{27}$. En todo caso, pareciera que en este tipo de hipótesis es ineludible admitir que la norma concluyentemente aplicable, aun cuando el juez la presente como la verdadera o la única ofrecida por el sistema, depende de un balance del respectivo valor de las normas involucradas. Por supuesto, si el problema se configurase como de tipo interpretativo y la teoría incorporase el mandato de interpretar coherentemente, precisando los conceptos en modo tal que se obtenga una única respuesta correcta, el sistema resultante sería siempre coherente. Pero ésta es la propuesta normativa de R. DWORKIN, que FERRAJOLI no acepta.

27 Por supuesto, esto no sucede si se entiende que las normas que pertenecen a la constitución son sólo aquellas que tienen el grado de generalidad explícitamente previsto en sus disposiciones. 
Por otra parte, si el constitucionalismo garantista acepta el pluralismo, no como un mero hecho digno de ser superado, sino como un valor que merece ser protegido por el propio Derecho, no se ve por qué se deba aceptar una reconstrucción en la que la posibilidad de antinomias entre normas del mismo nivel $-\mathrm{y}$ la respectiva necesidad de balances- que el pluralismo inevitablemente comporta no sea reconocida como tal.

Conforme a la propia práctica en la que están instaurados, los criterios últimos de validez, que normalmente permiten demarcar casos claros de normas excluidas e incluidas en el sistema, en situaciones cuantitativamente marginales —que son todas las que llegan a los tribunales-, pueden tener más de una interpretación y evaluación plausibles, contrastantes entre sí. En estas situaciones marginales el contenido o la relación axiológica existente entre las normas de la más alta jerarquía del sistema, puede ser objeto de balance o ponderación. Pero ello no significa que tales criterios no tengan, en general, un significado y un peso determinados. Por consiguiente, no justifica que sean caracterizados como pautas con significado o valor abiertos.

Es posible pensar que los casos de indeterminación de los criterios últimos confirman la tesis de que el contenido y/o la ordenación axiológica de los mismos, en tales casos, depende de las concretizaciones que realizan autoridades que los interpretan y aplican ${ }^{28}$. Sin embargo, esta conclusión no se sigue. Ciertamente, las mayorías políticas en un parlamento o congreso, mediante balances, deciden cómo interpretar los derechos, o cómo resolver los conflictos entre derechos respecto de ciertos tipos de casos. Y los jueces hacen lo mismo con respecto a casos individuales. Pero si pensamos en la sucesión de las autoridades políticas se ve claramente que la tesis de que ellas, mediante la legislación, concretan el contenido de la constitución y las jerarquías entre derechos lleva a conclusiones absurdas. Si éste fuese el caso, toda mayoría política estaría vinculada a la interpretación y/o jerarquía establecidas por la mayoría precedente puesto que, por hipótesis, ella habría ya especificado mediante sus leyes el contenido de la constitución. Es decir, una mayoría política no podría dictar leyes contrastantes con las de la mayoría precedente. La alternativa es pensar que cada legislatura, independientemente, determina a su arbitrio el contenido de la constitución. En este caso la constitución cambia de una legislatura a otra y el control de legitimidad constitucional de las decisiones de la autoridad política carece de sentido, dado que, por hipótesis, el contenido específico de la constitución es el que dicha autoridad política le confiere. En el primer caso, los jueces deberían permanentemente cuestionar la constitucionalidad de las normas dictadas por la mayoría política de turno ya que, muy probablemente, estarán en tensión con las emanadas de la mayoría precedente. En el segundo caso, sucedería exactamente lo contrario, estarían necesariamente vinculados a las decisiones de la mayoría política de turno sin poder cuestionar su legitimidad constitucional, puesto que, por hipótesis, cada mayoría política determina cómo ha de entenderse lo que la constitución permite o prohíbe.

28 Según algunos autores, la concretización o especificación es una de las vías para identificar estas normas fundamentales. Con respecto a la concretización realizada por los jueces, vid. G. PINO, Diritti e interpretazione, Bologna, Il Mulino, 2010, 103-106. Es interesante notar, también, que si estas normas, no derivadas, se identifican mediante abducción o, en todo caso, «de abajo hacia arriba», se cae irremediablemente en un problema de circularidad y se falsifica la tesis de que ellos son condiciones de validez de las restantes normas del sistema. En realidad dependen de ellas. 
En suma, parece ineludible aceptar que, conforme a la práctica, en ciertos casos puede no haber criterios para decidir los problemas de indeterminación o de conflictos de derechos fundamentales. Frente a este tipo de casos sucede algo parecido a lo que sucede, en general, frente a expresiones ambiguas o vagas. Al igual que quien usa este tipo de expresiones puede decidir el sentido en el que lo hace, las autoridades eligen y deciden el sentido preciso o la ordenación axiológica que confieren a los distintos derechos fundamentales. Pero esas decisiones, que efectivamente pueden cancelar la indeterminación respecto de los casos a los que pretenden referirse, no cancelan los otros sentidos u ordenaciones que ellos de hecho tienen. No está a disposición de quien los interpreta o aplica hacerlo. Y esto es lo mismo que decir que sus específicas decisiones no determinan su sentido o posición axiológica: la ambigüedad, o la indeterminación de su significado o valor permanecen. Justamente por este motivo es que podemos criticar esas decisiones y, a su vez, autoridades diferentes (o la misma autoridad en una ocasión diferente) pueden legítimamente interpretarlos y aplicarlos conforme a alguno de los otros sentidos u ordenaciones que ellos admiten. 